Original Research Article

\title{
Relevance of lung ultrasound in diagnosis of severe neonatal pneumonia
}

\author{
Dinakar $\mathbf{P}^{1}$, Bharat Reddy $\mathbf{D}^{2}$, Deepika $\mathbf{D}^{3}$, Mithila $\mathbf{M}^{4}$ \\ ${ }^{1}$ Dr Dinakar Prithviraj, Associate Professor, ${ }^{2}$ Dr. Bharath Reddy Assistant Professor, ${ }^{3}$ Dr. Deepika Dodda, Junior \\ Resident, ${ }^{4}$ Dr. Mithila das Mazumder, Junior Resident, all authors are affiliated with Department of Pediatrics, Vydehi \\ Institute of Medical Sciences and Research Institute, Bengaluru, Karnataka, India.
}

Address of Correspondence: Dr Dinakara Prithviraj, Associate Professor, Department of Pediatrics, Vydehi Institute of Medical Sciences, Bangalore. E-mail: drdinakar.nishanth@gmail.com

\begin{abstract}
Introduction: Neonatal pneumonia is a serious respiratory infection accounting for $10 \%$ of the neonatal deaths. Objective: The aim and objective of our study was to evaluate the accuracy of lung ultrasound to diagnose neonatal pneumonia. Methods: A 3-year prospective observational study was done between October 2013 to September 2016 in the neonatal ICU of Vydehi institute of medical sciences. About 86 neonates presented with features of pneumonia of which 21 cases had no $\mathrm{x}$ ray or ultrasonographic features. The control group comprised 65 neonates with no pulmonary disease are taken into the control group and compared. Results: 86 neonates were included in this study with clinical features of pneumonia of which 21 cases had no x rays or ultrasonographic features, so were not taken into the study group. Of the 65 cases included in the study; 39 were males and 26 females. In the 65 cases studied, 63 ( $96 \%$ ) had tachypnea, retractions in $42(64 \%)$ and grunting in $38(58 \%)$ of the cases. Three $(4.6 \%)$, of the studied cases died while the remaining 62(93\%) cases improved and were discharged. Conclusion: In conclusion, the study confirms that lung ultrasonography is useful in the diagnosis of neonatal pneumonia.
\end{abstract}

Keywords: Neonatal pneumonia, Respiratory Distress, Lung Ultrasonography

\section{Introduction}

Neonatal pneumonia is a serious respiratory infection caused by various bacteria and less likely viral leading to high morbidity and mortality. It is estimated that $10 \%$ of the neonatal deaths accounts for neonatal pneumonia in the neonatal ICU in the developing countries. Pneumonia may be acquired by intrauterine, intrapartum or postnatal-community or nosocomial causes. The pathogens include mainly bacteria, followed by viruses and fungi which induce inflammatory changes in the lungs [1].

This may cause epithelial injury to the airways, leakage of proteinaceous fluid into the alveoli and interstitium, leading to surfactant deficiency or dysfunction. Important predisposing factors in the evolution of pneumonia are immaturity, low birth weight, premature rupture of membranes, chorioamnionitis and factors associated with prolonged neonatal intensive care.

Manuscript received: $7^{\text {th }}$ February 2017

Reviewed: $15^{\text {th }}$ February 2017

Author Corrected: $21^{\text {st }}$ February 2017

Accepted for Publication: 28 ${ }^{\text {th }}$ February 2017
Depending on the time of manifestation of infection neonatal pneumonia may be classified as early onset pneumonia (within the first 3 days of life, mostly within 48 hours), or late onset pneumonia (within 4 and 28 days of life) [2]. Congenital or intrauterine pneumonia can be considered a variant of early onset pneumonia. Clinical signs are non specific and present as respiratory distress of various degree, suspicious appearing tracheal aspirates, cough, apnea, high or low temperature, poor feeding, abdominal distension, and lethargy. Tachypnea is a predominant clinical sign, present in $60-89 \%$ of cases. Persistent fever is rather unusual, but has been reported in neonates with viral pneumonia [3].

In the neonatal intensive care unit (NICU), bedside lung auscultation and chest radiography are routinely performed on a daily basis for assessing lung status in infants with chest problems. Interpretation of the location and nature of an area of increased opacity on chest radiographs is sometimes problematic, particularly in young infants with varied configurations 
of the normal thymus, and differentiation between pulmonary, pleural, and mediastinal lesions is not always easy. Because of many advantages such as the absence of radiation exposure, non-invasiveness, low cost, safety, and ready avail- ability, transthoracic ultrasonography (TUS) represents an emerging and useful technique in the management of pleural and pulmonary diseases [4]. In 1986 Weinberg et al. (1986) described a new method of evaluating CAP by the use of lung ultrasonography (LUS). Numerous subsequent studies have shown that it is an accurate, reliable and radiation-free tool in the diagnosis of pneumonia. The aim and objective of our study was to evaluate the accuracy of lung ultrasound to diagnose neonatal pneumonia $[5,6]$.

\section{Materials and Methods}

A 3-year prospective observational study was done between October 2013 to September 2016 in the neonatal ICU of Vydehi institute of medical sciences. The institutional review board of the Vydehi institute of medical sciences approved the study protocol. A total of 867 neonates were admitted to the Neonatal ICU during this study period of which 86 neonates presented with features of pneumonia. Neonates presenting with clinical picture of pneumonia were enrolled in the study. Only neonates presenting after $48 \mathrm{~h}$ of life were included as an attempt to exclude the possibility of Respiratory Distress Syndrome (RDS) and Transient Tachypnea of the Newborn (TTN). Children with congenital heart disease, pulmonary hemorrhage or pneumothorax were excluded from the study.

The neonatologist in ICU with the following criteria diagnosed neonatal pneumonia:

(1) Presence of cough, fever, or dyspnea

(2) Fine crepitation on auscultation

(3) Signicantly increased or decreased WBC count, increased neutrophils or ratio of immature/total neutrophils or high C-reactive protein level

(4) Patchy, blurred shadows with uneven density in the lung fields on chest radiograph.

Neonates with congenital heart disease (CHD) were excluded from the study to rule out pulmonary edema secondary to congenital heart disease. The neonates suspected to have pneumonia were subjected, history taking to include sex, gestational age, postnatal age, mode of delivery, weight, presenting symptoms and their onset. A full clinical examination was performed to include general, cardiac, abdominal and chest examination. Signs of respiratory distress (respiratory rate, presence of retractions, grunting or cyanosis) were observed and chest auscultation was done. The same investigator performed auscultation immediately before performing the US scanning. Twelve lung regions were systematically examined: the upper and lower parts of the anterior, lateral, and posterior regions of the left and right chest walls with the patient in the supine position. Abnormal auscultatory findings include diminished breath sounds, presence of bronchial breath sounds and presence of fine crepitations. The extent of lung injury was assessed as the number of regions where auscultation was abnormal.

The control group comprised 65 neonates with no pulmonary disease who were hospitalized for noninfectious jaundice, premature birth, or other nonpulmonary conditions. Chest $\mathrm{X}$ ray was not done in the control group. Laboratory investigations including complete blood count with differential, $\mathrm{C}$ reactive protein, blood culture and endotracheal aspirate in ventilated cases were done. Chest radiography with the patient in the supine position were obtained using a Philips Mobile Medical X-ray system before US scanning that was read by the same radiologist. Exposure time, focus-film distance, and degree of exposure were standardized for each patient to obtain the best radiographic quality. Lung parenchyma was divided into 12 regions by a cephalo caudal midaxillary line and a transversal hilar line. Upper lung regions were defined as lung regions delineated by the apex, mid-axillary line, mediastinal line, and hilar line. Upper and lower lateral lung regions were defined as lung regions delineated by the external limit of the chest wall, mid-axillary line, and apex (upper) or diaphragm (lower). Upper and lower posterior lung regions were defined as lung regions with radiologic signs erasing the mediastinum border ("silhouette sign") and delineated by the mediastinum, mid-axillary line, hilar line, and apex (upper) or diaphragm (lower). The extent of lung injury was assessed as the number of lung regions with radiologic signs suggestive of alveolar consolidation.

Lung ultrasonography was performed immediately after the chest radiography, with a Philips HD 11XE Diagnostic Ultrasound System, with a linear 3-12 MHz probe. Antero- lateral parts of the chest wall were examined with the patient in the supine position, whereas posterior parts were examined with the patient in the lateral position. Lung ultrasound examinations were also performed for a control group of 65 healthy neonates to determine a normal ultra- sound lung 
pattern. Trans sternal, parasternal, and intercostal approaches are good for imaging of the lung, pleura, and anterior mediastinum. Sector transducers are used in subxiphoid and Trans diaphragmatic approaches with the liver used as the acoustic window for evaluating juxta phrenic paravertebral lesions. Suprasternal and supraclavicular approaches facilitate evaluation of the upper mediastinum and lung apexes. US are performed in the supine, prone, or decubitus position. Images are obtained in the transverse, longitudinal, and inclined transverse or inclined longitudinal planes to maximize demonstration of the lesion. Color flow imaging may be helpful in characterizing the lesion by demonstrating the vascularity and flow pattern and in searching for anomalous vessels, such as those that occur in pulmonary sequestration. The same radiologist did all scans.

Statistical analysis: Data analyses were performed with SPSS for Windows version 16.0 (IBM) software. Abnormal ultrasound findings were compared between the two study groups by Fisher exact test, and the specificity and sensitivity of the findings for diagnosing neonatal pneumonia were calculated.

\section{Results}

About 86 neonates were included in this study with clinical features of pneumonia of which 21 cases had no X rays or ultrasonographic features, so were not taken into the study group.

Table-1: General clinical information.

\begin{tabular}{|c|c|c|c|c|c|}
\hline Group & Sex (M/F) & $\begin{array}{c}\text { Day of admission } \\
(\text { Mean } \pm \text { SD) }\end{array}$ & Weight & $\begin{array}{c}\text { Term/ } \\
\text { Preterm }\end{array}$ & $\begin{array}{c}\text { Vaginal/ } \\
\text { C Section }\end{array}$ \\
\hline Pneumonia (N=65) & $39 / 26$ & $\begin{array}{c}3-18.5 \\
(7.2 \pm 2.3)\end{array}$ & $2723 \pm 657 \mathrm{gms}$ & $47 / 18$ & $43 / 22$ \\
\hline Control $(\mathrm{N}=65)$ & $37 / 27$ & - & $2895 \pm 584 \mathrm{gms}$ & $40 / 25$ & $48 / 17$ \\
\hline
\end{tabular}

Table 1 showing 65 cases included in the study; 39 were males and 26 females, their postnatal ages on admission ranged from 3 to 18.5 days with a mean ( \pm standard deviation (SD)) of $7.2 \pm 2.3$ days. Their mean weight was $2723 \pm 657 \mathrm{gms}$ and mean gestational age was 37 weeks. Forty-three cases were delivered vaginally $(66.1 \%)$ and $22(33.8 \%)$ were delivered by caesarian section. Out of 65 neonates with pneumonia 18(27.6\%) were Preterms.

Table-2: Clinical profile of the studied cases.

\begin{tabular}{|c|c|c|c|}
\hline Pneumonia features & $\begin{array}{l}\text { Ultrasound findings } \\
\text { Not present }(n=21)\end{array}$ & $\begin{array}{l}\text { Ultrasound findings } \\
\text { Present }(n=65)\end{array}$ & Total \\
\hline $\begin{array}{c}\text { Chest } X \text { ray findings not present } \\
\text { Number } \\
\% \\
\end{array}$ & $\begin{array}{c}21 \\
(24 \%) \\
\end{array}$ & $\begin{array}{c}9 \\
(10.4 \%) \\
\end{array}$ & $\begin{array}{c}30 \\
(34 \%) \\
\end{array}$ \\
\hline Chest $\mathrm{X}$ ray present & $\begin{array}{c}0 \\
(0)\end{array}$ & $\begin{array}{c}56 \\
(65 \%)\end{array}$ & $\begin{array}{c}56 \\
(65 \%)\end{array}$ \\
\hline $\begin{array}{c}\text { Number } \\
\%\end{array}$ & $\begin{array}{c}21 \\
(24 \%)\end{array}$ & $\begin{array}{c}65 \\
(75 \%)\end{array}$ & $\begin{array}{c}86 \\
(100 \%)\end{array}$ \\
\hline
\end{tabular}

Table 2 showing 65 cases studied, 63 (96\%) had tachypnea, retractions in 42(64\%) and grunting in 38(58\%) of the cases.

Table-3: Chest X-ray and ultrasonography cross-tabulated findings of 86 clinical suspected cases of pneumonia.

\begin{tabular}{|c|c|c|}
\hline Sign & Number & \% \\
\hline Tachypnea $(>60$ breaths/min) & 63 & 64 \\
\hline Retractions & 42 & 58 \\
\hline Grunting & 38 & 27 \\
\hline Cyanosis & 18 & 50 \\
\hline Decreased air entry & 33 & 58 \\
\hline Fine crepitations & 38 & 29 \\
\hline Bronchial breathing & 19 & \\
\hline
\end{tabular}


Table 3 shows out of 86 cases 65 had chest x ray or ultrasound features of pneumonia and 21 cases of clinical suspected of pneumonia had neither chest x ray nor USG findings so excluded from study. All the 65 cases had ultrasound features of pneumonia and Chest X-ray findings were not present in 9 cases.

Table-4: Occurrence Rate of Several Common Ultrasound Signs of Pneumonia.

\begin{tabular}{|c|c|c|c|}
\hline Ultrasound sign & Pneumonia group (n=65) & Control group (n=65) & P value \\
\hline Lung consolidation & $65(100 \%)$ & $0(0)$ & $<0.001$ \\
\hline Dynamic air bronchograms $^{\mathrm{A}}$ & $29(44 \%)$ & $0(0)$ & $<0.001$ \\
\hline Pleural line abnormalities & $51(82 \%)$ & $0(0)$ & $<0.001$ \\
\hline Pleural effusion & $14(21 \%)$ & $0(0)$ & $<0.001$ \\
\hline
\end{tabular}

\section{${ }^{A}$ means coarse appearance, irregularity and disappearance}

Table 4 shows USG revealed pneumonic patches in 65 patients (75\%) that appear as oblong or wedge shaped sub pleural isoechoic areas, branching or scattered echogenic bright dots that represent the air bronchograms were seen within these areas. Color Doppler imaging revealed the branching pattern of the pulmonary vessels within the consolidated segment. Nine (10.1\%) had free US examinations. Of the 65 patients with US detected pneumonic consolidation, 56 patients had pneumonic patches detected by both US and chest X-ray. Of 65 cases with USG features of consolidation 29(44\%) had air bronchograms, $14(21 \%)$ had pleural effusion, pleural line abnormalities in 51(65\%) and interstitial syndrome $44(67 \%)$.

Two patients had radiographically suspected atelectasis; USG confirmed these findings demonstrating crowdedness of the echogenic air bronchograms. The five neonates diagnosed clinically as pneumonia (with 1 or 2 positive sepsis screening laboratory parameter) with no significant findings in the X-ray and ultrasonography showed improvement with antibiotic therapy and their course followed. Three (4.6\%), of the studied cases died while the remaining 62(93\%) cases improved and were discharged.

\section{Discussion}

Pneumonia is the leading cause of death in neonatal ICU in developing countries. The diagnosis of pneumonia has been highly dependent upon imaging since physical examination had lacunae in detection of pneumonia and early and effective detection was vital in detection the duration of ICU stay and mortality. The need to rule out pneumonic process is important in the NICU, so there is a continuous concern about the balance between the diagnostic value and the potential harmful effects of chest X-rays frequently done for neonates in NICU [7].

In our study, the usefulness of lung USG for diagnosing neonatal pneumonia showed large areas of consolidation with irregular margins and air bronchograms, pleural line abnormalities, and interstitial syndrome and prediction of the cases more early and effective than chest radiograph. In the present study, lung consolidation in neonatal pneumonia had the following ultrasonographic features like a large area of consolidation, irregular margins around the area of consolidation and dynamic air bronchograms. In the present study, air bronchograms (44\%), interstitial syndrome (93\%) and pleural line abnormalities (82\%) were observed, but nonspecific ultrasound findings in neonatal pneumonia and are related to inflammatory changes [8].

In 9 neonates with USG diagnosed pneumonic patches not seen in their chest X-rays, however these X-rays were not considered normal since apparent thickening of pulmonary interstitium, and hyper inflated lungs were noted not considered for chest infection. Hence, combining USG with chest X-rays would be more accurate in ruling out the presence of underlying pneumonic process. Although radiological findings are commonly accepted as the gold standard for defining pneumonia, there are no validated definitions for X-ray interpretation. Also, there is a significant variation in intra and inter observer agreement among radiologists on the interpretation of the same chest X- ray images and on the radiographic features used for diagnosis of pneumonia

In the study, tachypnea was a main presenting feature in $96 \%$ of cases and retractions were present in majority 
$64 \%$ of studied cases. Similarly, retractions and tachypnea were the main presenting signs in neonatal pneumonia for retractions and $88.3 \%$ and $64.3 \%$ for tachypnea and retractions, respectively in the study done by Marthur and colleague. Diagnosis of neonatal pneumonia on clinical basis remains of superior value. In developing countries, childhood pneumonia is diagnosed using clinical parameters, usually based on the presence of cough and raised respiratory rate [9]. This is a sensitive definition, maximizing the number of children identified and treated empirically, but it is nonspecific

\section{Conclusion}

In conclusion, the study confirms that lung ultrasonography is useful in the diagnosis of neonatal pneumonia. Ultrasonography is accurate, inexpensive, and easy to perform at the bedside, making it especially suitable for neonates. Lung ultrasonography may also be useful for guiding life-saving therapies in emergency situations. Most importantly, ultrasonography does not expose the patient to radiation, in contrast to radiography and CT imaging, which carry a risk of DNA damage and are, therefore, associated with an increased risk of cancer. Bedside lung ultrasound examination is both feasible and convenient in the neonatal ward and may eventually replace chest radiography.

Funding: Nil, Conflict of interest: None initiated, Perission from IRB: Yes

\section{References}

1. Reissig A, Kroegel C. Transthoracic sonography of diffuse parenchymal lung disease: the role of comet tail artifacts. J Ultrasound Med. 2003 Feb;22(2):173-80.

2. Wilson-Costello D, Rao PS, Morrison S, Hack M. Radiation exposure from diagnostic radiographs in extremely low birth weight infants. Pediatrics. 1996 Mar; 97(3):369-74.

3. Yang PC, Luh KT, Chang DB, Yu CJ, Kuo SH, Wu HD. Ultrasonographic evaluation of pulmonary consolidation. Am Rev Respir Dis. 1992 Sep;146 (3):757-62.

4. Blaivas M. Lung ultrasound in evaluation of pneumonia. J Ultrasound Med. 2012 Jun;31(6):823-6.

5. Cortellaro F, Colombo S, Coen D, Duca PG. Lung ultrasound is an accurate diagnostic tool for the diagnosis of pneumonia in the emergency department. Emerg Med J. 2012 Jan;29(1):19-23. doi: 10.1136/emj. 2010.101584. Epub 2010 Oct 28.

6. Volpicelli G, Elbarbary M, Blaivas $M$, et al. International Liaison Committee on Lung Ultrasound (ILC-LUS) for International Consensus Conference on Lung Ultrasound (ICC-LUS). International evidencebased recommendations for point-of-care lung ultrasound. Intensive Care Med. 2012 Apr;38(4):57791. doi: 10.1007/s00134-012-2513-4. Epub 2012 Mar 6.

7. Cattarossi L. Lung ultrasound: its role in neonatology and pediatrics. Early Hum Dev. 2013 Jun;89 Suppl 1:S17-9. doi: 10.1016/S0378-3782(13)70006-9.

8. Liu J. Lung ultrasonography for the diagnosis of neonatal lung disease. J Matern Fetal Neonatal Med. 2014 May; 27 (8): 856 - 861. doi: 10.3109 /14767058. 2013.844125. Epub 2013 Oct 17

9. Chen SW, Fu W, Liu J, Wang Y. Routine application of lung ultrasonography in the neonatal intensive care unit. Medicine (Baltimore). 2017 Jan;96(2):e5826. doi: 10.1097/MD.0000000000005826.

\section{How to cite this article?}

Dinakar P, Bharat Reddy D, Deepika D, Mithila M. Relevance of lung ultrasound in diagnosis of severe neonatal pneumonia. J PediatrRes.2017;4(02):128-132.doi:10.17511/ijpr.2017.i02.07. 